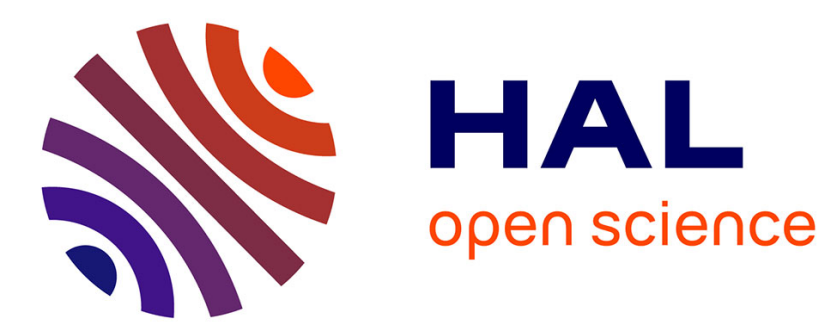

\title{
Observavility Brunovsky Normal Form: Multi-Output Linear Dynamical Systems
}

Driss Boutat, Frédéric Kratz, Jean-Pierre Barbot

\section{To cite this version:}

Driss Boutat, Frédéric Kratz, Jean-Pierre Barbot. Observavility Brunovsky Normal Form: MultiOutput Linear Dynamical Systems. American Control Conference, ACC09, IEEE, Jun 2009, St. Louis, MO, United States. hal-00772281

\section{HAL Id: hal-00772281 \\ https://hal.inria.fr/hal-00772281}

Submitted on 10 Jan 2013

HAL is a multi-disciplinary open access archive for the deposit and dissemination of scientific research documents, whether they are published or not. The documents may come from teaching and research institutions in France or abroad, or from public or private research centers.
L'archive ouverte pluridisciplinaire HAL, est destinée au dépôt et à la diffusion de documents scientifiques de niveau recherche, publiés ou non, émanant des établissements d'enseignement et de recherche français ou étrangers, des laboratoires publics ou privés. 


\title{
Observavility Brunovsky Normal Form: Multi-Output Linear Dynamical Systems
}

\author{
Driss Boutat, Frédéric Kratz and Jean-Pierre Barbot
}

\begin{abstract}
This paper gives the sufficient and necessary conditions to guarantee the existence of a linear change of coordinates to transform a multi-output linear dynamical system (modulo a nonlinear term depending on inputs and outputs) in the observability Brunovsky canonical form.
\end{abstract}

\section{INTRODUCTION}

For a single output dynamical linear system the observability rank condition is a necessary and sufficient condition to transform it into the Brunovsky observability normal form. In this last form, it is possible to use classical observer such that [8] observer and, [5] observer. For multi-input linear dynamical systems these condition were given by [3]. For multi-output linear dynamical systems, to our knowledge, there does not exist in literature an equivalent result. However, for multi-output nonlinear dynamical systems there are several works on the topic. All this works dealt with the so-called : Observer linearization error dynamic. This last notion was first addressed by [6] for dynamical systems with a single output. [7] gave, for a multi-output nonlinear system, the sufficient conditions to solve the observer linearization error problem even with a diffeomorphism on the outputs.

[11] gave the sufficient and necessary conditions to solve the linearization problem for multi-output nonlinear systems. After these two important works in the field of observers, other interesting research was followed, we can quote for examples: [1], [2], [4], [10].

Even if the results, obtained in the works quoted above, can be used for multi-output linear dynamical systems. In this paper, we will provide in an algebraic way the necessary and sufficient conditions which ensure existence of a linear change of coordinates to transform a multi-output linear system into the Brunovsky normal form. This paper is outlined as follows. In the next section, we give the notations and the problem statement. In section 3 , we recall well-known facts about a matrix and deduce a first preliminary result. In section 4 , we will state our main result. In section 5 , we provide an algorithm to compute the linear change of coordinates.

D. Boutat and F. Kratz are with ENSI de Bourges, Institut PRISME, 88 Boulevard de Lahitolle, 18020 Bourges, France. driss.boutatfrederic.kratzeensi-bourges.fr.

J.P. Barbot is with ECS/ENSEA, 6 Avenue du Ponceau, 95014 CergyPontoise, France. barboteensea.fr

\section{NOTATION AND PROBLEM STATEMENT}

Consider the following multi-output dynamical system:

$$
\begin{aligned}
\dot{x} & =A x+\gamma(y, u) \\
y & =C x
\end{aligned}
$$

where:

- $x \in R^{n}$ are the state variables, $y=\left(y_{i}\right)_{1 \leq i \leq m}$ are the outputs and $u$ is an input,

- $A$ is a $n \times n$ matrix and $\gamma$ is a vector depending on input and outputs

- $C=\left[\begin{array}{c}C_{1} \\ C_{2} \\ . . \\ C_{m}\end{array}\right]$

is a $m \times n$ matrix with linearly independent components.

Before, to state the problem to solve here, let us recall the socalled observability indices for dynamical systems (1-2). For this, without loss of generality, we will assume throughout this paper the following.

We assume that the pair (1-2) is observable thus, the following observability rank condition:

$\operatorname{rank}\left[\begin{array}{c}C \\ C A \\ . . \\ C A^{n-1}\end{array}\right]=n$

is fulfilled.

Now, let us define $s_{0}=\operatorname{rank}[C]=m$, and by induction:

$\mathrm{s}_{k}=\operatorname{rank}\left[\begin{array}{c}C \\ C A \\ \ddot{A} A^{k-1}\end{array}\right]-s_{k-1}$.

The following definition is drawn from [9].

Definition 1: We call observability indices of the dynamical system (1-2) the integers given for $1 \leq s \leq m$ by: $\mathrm{r}_{i}=\operatorname{card}\left\{s_{j} \geq i\right.$ for $\left.j \geq 0\right\}$.

By definition, we have $r_{1} \geq r_{2} \geq \ldots \geq r_{m}$ and from the observability rank condition II, we have obviously:

$\mathrm{r}_{1}+\ldots+r_{m}=n$.

By reordering the outputs $\left(y_{i}\right)_{1 \leq i \leq m}$, we can assume that for $1 \leq i \leq m$ output $y_{i}$ has index $r_{i}$. Thus we have: 


$$
\left[\begin{array}{c}
{\left[\begin{array}{c}
C_{1} \\
\vdots \\
C_{m}
\end{array}\right]} \\
C_{1} A \\
. \cdot \\
C_{1} A^{r_{1}-1} \\
C_{2} A \\
. . \\
C_{2} A^{r_{2}-1} \\
. . \\
C_{i} A \\
. . \\
C_{i} A^{r_{i}-1}
\end{array}\right]=r_{1}+r_{2}+\ldots+r_{i}+m-i,
$$

The problem that we will answer here can be stated as follows.

Problem 1: Find the necessary and sufficient algebraic conditions for the existence of a linear change of coordinates $z=M x$ such that the dynamical system (1-2) becomes:

$$
\begin{aligned}
& \dot{z}_{i}=A_{i} z_{i}+\beta_{i}\left(u, z_{1, r_{1}}, \cdots, z_{1, r_{m}}\right) \\
& z_{1, r_{1}}=y_{1} \\
& z_{i, r_{i}}=y_{i}+\sum_{j=1}^{i-1} \alpha_{i, j} y_{j} \quad \text { for } \quad 2 \leq i \leq m \\
& \text { where } A_{i}=\left[\begin{array}{cccc}
0 & \cdots & 0 & 0 \\
1 & \cdots & 0 & 0 \\
\vdots & \ddots & \vdots & \vdots \\
0 & \cdots & 1 & 0
\end{array}\right]_{r_{i} \times r_{i}} \text {, } \\
& \text { and } z_{i}=\left(z_{i, j}\right)_{1 \leq j \leq r_{i}} \text { for } 1 \leq i \leq m \text {. }
\end{aligned}
$$

We will say that a dynamical system under (5-7) form is in the Brunovsky observability canonical normal form modulo an input-output injection.

In the (5-7) form, we can use the well-known Lunberger observer with nonlinear input and output terms:

$$
\dot{\widehat{z}}_{i}=A_{i} \widehat{z}_{i}-K(\bar{y}-\widehat{\bar{y}})+\beta_{i}\left(u, \widehat{z}_{1, r_{1}}, \cdots, \widehat{z}_{1, r_{m}}\right)
$$

where for all $1 \leq i \leq m$ we set $\bar{y}_{i}=z_{i, r_{i}}$ and $K$ is the gain.

Remark 1: If the case of system (1-2) without noise is perfectly resolved by 8 it is not the case for noisy systems For example, if the output measurement is corrupted by the noise and the output acts quadratically in term then a bias appears.

\section{MAThematical BACKGRound}

In this section, we recall some facts about the structure of a given $n \times n$ matrix $\Im$.

A matrix $\Im$ is said to be cyclic if there exists a vector $v \in R^{n}$ such that the following family:

$\left\{\mathrm{v}, \ldots, \Im^{n-1} v\right\}$ is a basis of $R^{n}$. This last fact is also equivalent to saying that the minimal polynomial of $\Im$ is equal to its characteristic polynomial.

Therefore, for a cyclic matrix $\Im$, if we denote its transpose by $A=\Im^{T}$ then, for $A$ we need only to have a single output $y=C x$ where $C=v^{T}$ such that the pair $(A, C)$ fulfils the observability rank condition. Thus,

$$
\operatorname{rank}\left[\begin{array}{c}
C \\
C A \\
. . \\
C A^{n-1}
\end{array}\right]=n .
$$

In this case, the following linear change of coordinates transforms the dynamical system (1-2) into the form (5-7) $(m=1)$ :

$$
\begin{aligned}
z_{n} & =C x \\
z_{n-k} & =C A^{k} x+\sum_{i=1}^{k} p_{n-i} C A^{k-i} x \text { for } 1 \leq k \leq n-1
\end{aligned}
$$

where $\left(p_{i}\right)_{0<i<n-1}$ are the coefficients of the characteristic polynomial of $A$ :

$$
P_{A}(s)=p_{0}+p_{1} s+\ldots+p_{n-1} s^{n-1}+s^{n}
$$

and the vector field $\beta(y, u)=\left(\beta_{i}(y, u)\right)_{1 \leq i \leq n}$ is given by:

$$
\begin{aligned}
\beta_{n} & =-p_{n-1} y+C \gamma(y, u) \\
\beta_{n-k} & =-p_{n-(k+1)} y \\
& +\sum_{i=1}^{k} p_{n-i} C A^{i-1} \gamma(y, u)+C A^{k} \gamma(y, u) \text { for } 1 \leq k \leq n-1
\end{aligned}
$$

To finish, take the derivative of $z_{1}$ given in (11), we obtain:

$$
\dot{z}_{1}=C A^{n} x+\sum_{i=1}^{n-1} p_{n-i} C A^{n-i} x+\varphi(y, u) .
$$

Therefore, by Cayley-Hamilton's theorem, we deduce from (12) expression that:

$$
\dot{z_{1}}=-p_{0} y+\varphi(y, u)=\beta_{1}(y, u) .
$$

Now, if a matrix $\Im$ is not cyclic, then we need more outputs to have the observability rank condition. Precisely, if the Frobenius normal form or rational canonical form of $A$ contains $p$ blocs then we need at least $m \geq p$ outputs to have the observability rank condition. Indeed, assume that the matrix

$$
A=\operatorname{bloc} \operatorname{diag}\left[F_{1}, . ., F_{m}\right]
$$

where $F_{i}$ for $1 \leq i \leq m$ are the Companion matrices. The characteristic polynomial $\mathcal{M}_{1}$ of $F_{1}$ is the minimal polynomial of $A$, and for $2 \leq i \leq m$ the characteristic polynomial $\mathcal{M}_{i}$ of the bloc $F_{i}$ devised the characteristic polynomial $\mathcal{M}_{i-1}$ of the bloc $F_{i-1}$ such that:

$$
P_{A}=\mathcal{M}_{1} \ldots \mathcal{M}_{p}
$$

Moreover, if we denote by $r_{i}$ the degree of $\mathcal{M}_{i}$, then $r_{1}+. .+r_{m}=n$. 
It is well-known that there exists a family of vectors $\left\{v_{i, 1}\right.$ for $\left.1 \leq i \leq m\right\}$ such that the family $\left\{v_{i, j}=\right.$ $B^{j} v_{i, 1}$ for $1 \leq i \leq m$ and $\left.0 \leq j \leq r_{i}-1\right\}$ is a basis of $R^{n}$. If we set

$$
E_{i}=\operatorname{span}\left\{v_{i, j} \quad \text { for } \quad 0 \leq j \leq r_{i}-1\right\}
$$

then it is a $A$-invariant vector subspace. Thus, $A E_{i} \subseteq E_{i}$ and we have:

$$
R^{n}=E_{1} \oplus \ldots \oplus E_{m}
$$

Let us sst

$$
C_{i}=v_{i, 1}^{T} \quad \text { for } \quad 1 \leq i \leq m
$$

then, we have the following preliminary result:

Claim 1: ideal case

Assume $A$ is cyclic and the output $y_{i}=C_{i}=v_{i, 1}^{T}$, then there exists a linear change of coordinates which transforms the dynamical system (1-2) into the form (5-7) with $\alpha_{i, j}=0$ in the relation (7).

Proof: For $1 \leq i \leq m$ we have $\Im E_{i} \subseteq E_{i}$ which implies that:

$$
C_{i} A^{r_{i}}=\sum_{j=1}^{r_{i}} p_{i, j} C_{k} A^{j-1}
$$

This last equation plays the part of Cayley-Hamilton's theorem in the single output $m=1$. Therefore the linear change of coordinates is given as in the beginning of this section for $m=1$. For each $1 \leq i \leq m$, we set:

$$
\begin{aligned}
z_{i, r_{i}} & =C_{i} x \quad \text { and, for } 1 \leq k \leq r_{i}-1 \\
z_{i, r_{i}-k} & =C A^{k} x+\sum_{j=1}^{k} p_{i, n-j} C A^{k-j} x
\end{aligned}
$$

\section{General CASE}

In this section, we assume that the number $m$ of the outputs is great or equal to the number $p$ of Frobinus blocs of the matrix $A$. As above $\left(r_{i}\right)_{1 \leq i \leq m}$ are the observability indices of (1-2). Under assumption II and relations (3-4), we have the following preliminary result.

Lemma 1: There exists a linear change of coordinates which transforms the dynamical systems (1-2) into the following form:

$$
\begin{aligned}
\dot{z}_{i}= & A_{i} z_{i}+\beta_{i}\left(u, z_{1, r_{1}}, \cdots, z_{1, r_{m}}\right) \\
z_{1, r_{1}}= & y_{1} \\
z_{i, r_{i}}= & y_{i}-\sum_{j=1}^{i-1} \sum_{k=1}^{r_{j}-r_{i}} p_{i, j, r_{j}-k} C_{j} A^{r_{j}-r_{i}-k} x \\
& \quad \text { for } \quad 2 \leq i \leq m
\end{aligned}
$$

Proof: For sake of clarity, we will give the proof for $m=2$. Recall from equation (4) that we have:

$$
\begin{array}{r}
C_{1} A^{r_{1}}=\sum_{j=1}^{r_{1}-1} p_{1,1, j} C_{1} A^{j-1}+q_{1,2} C_{2} \\
C_{2} A^{r_{2}}=\sum_{j=1}^{r_{1}-1} p_{2,1, j} C_{1} A^{j-1}+\sum_{j=1}^{r_{2}-1} p_{2,2, j} C_{2} A^{j-1} .
\end{array}
$$

Now, it is easy to see that the following change of coordinates:

$$
\begin{aligned}
z_{1, r_{1}} & =C_{1} x \\
z_{1, r_{1}-k} & =C_{1} A^{k} x-\sum_{j=1}^{k} p_{1, j, r_{1}-j} C_{1} A^{k-j} x,
\end{aligned}
$$

and

$$
\begin{aligned}
z_{2, r_{2}} & =C_{2} x-\sum_{j=1}^{r_{1}-r 2} p_{2, r_{1}-j} C_{1} A^{j-1} x \\
z_{2, r_{2}-k} & =C_{2} A^{k} x-\sum_{j=1}^{k} p_{2, j, r_{i}-j} C_{2} A^{k-j} x \\
& -\sum_{j=1}^{r_{1}-r 2} p_{2,1, r_{1}-j} C_{1} A^{k+j-1} x
\end{aligned}
$$

do the work.

Remark 2: The dynamics (15) in lemma 1 are in the form sought in (5). However, the outputs are not in the form of (5). Indeed, for $2 \leq i \leq m$ the $i^{t h}$ output $y_{i}$ depends on the formers output $y_{1}, \ldots, y_{i-1}$ and some of their derivatives as we saw it in formula (17).

Now, we can deduce the following result from lemma 1.

Corollary 1: Assuming hypothesis II and relation (3) then there exists a linear change of coordinates which solves the problem 1 if and only if equation 4 is in the following form:

$$
C_{i} A^{r_{i}}=\sum_{k=1}^{i} \sum_{j=1}^{r_{i}} p_{i, k, j} C_{k} A^{j-1}+\sum_{k=i+1}^{m} q_{i, k} C_{k} .
$$

Thus, for $2 \leq i \leq m$ we have $p_{i, k, j}=0$ for all $2 \leq k \leq i$ and $j>r_{i}$.

\section{CHANGE OF COORDINATES}

In this section, we will give the algorithm to compute the change of coordinates. Let the following family of vectors $\left\{\tau_{1, i}\right\}_{1 \leq i \leq m}$ defined by the following algebraic equations :

$$
\begin{array}{r}
C_{j} A^{k-1} \tau_{i, 1}=0 \text { for } j<i \text { and } 1 \leq k \leq r_{i} \\
C_{i} A^{k-1} \tau_{i, 1}=0 \quad \text { for } 1 \leq k \leq r_{i} \\
\\
C_{i} A^{r_{i}-1} \tau_{i, 1}=1 \\
C_{j} A^{k-1} \tau_{i, 1}=0 \text { for } j>i \text { and } 1 \leq k \leq r_{j} .
\end{array}
$$

Now, we set for all: $\tau_{j, i}=C_{i} A^{j} \tau_{1, i}$ for $1 \leq i \leq$ $m$ and $1 \leq j \leq r_{i}-1$. By the observability rank condition the family $\left\{\tau_{j, i}\right\}$ is a basis of $R^{n}$. Let 
$\theta=\left[\begin{array}{c}C_{1} \\ C_{1} A \\ \ddot{A_{1}-1} \\ C_{2} \\ \ddot{C_{m}} \\ . . \\ C_{m} A^{r_{m}-1}\end{array}\right]$ and let

Now set $M=\Lambda^{-1} \theta$

Lemma 2: The change of coordinates $z=M x$ transforms the dynamical system (1-2) into the form given in lemma 1 .

Proof: Let us set for $1 \leq i \leq m$ for $1 \leq j \leq r_{i}$ : $\mathbf{M} \tau_{i, j}=\frac{\partial}{\partial z_{i, j}}$. Now, take the derivative in the direction $\frac{\partial}{\partial z_{i, j}}$ of $\dot{z}=M \dot{x}$. We obtain for $1 \leq i \leq m$ for $1 \leq j \leq r_{i}-1$ :

$$
\begin{aligned}
\frac{\partial}{\partial z_{i, j}} M \dot{x} & =M A \tau_{i, j} \\
& =M \tau_{i, j+1}=\frac{\partial}{\partial z_{i, j+1}} .
\end{aligned}
$$

Therefore, by integration, the dynamic (1) in the new coordinates has the form (5) and the outputs have the form (17).

Now, we will give the equivalent condition (18) of corollary 1 by means of the vectors $\tau_{i, j}$.

Corollary 2: Condition (18) is equivalent to the following: for all $1 \leq i \leq m-1$ such that $j<i$ and $r_{j}>r_{i}$

$$
C_{j} A^{k} \tau_{i, 1}=0 \text { for } r_{j}-r_{i}<k \leq r_{j}
$$

Remark 3: Condition (23) means that for $2 \leq i \leq m$ output $y_{i}$ do not depend on the derivatives of outputs $y_{j}$ for $j<i$.

\section{DISCUSSION ON THE NONLINEAR DYNAMICAL SYSTEMS}

Consider a multi-output nonlinear dynamical system in the following form:

$$
\begin{aligned}
\dot{x} & =f(x) \\
y & =h(x) .
\end{aligned}
$$

where $f$ is a smooth vector field and $h$ a smooth function with $m$-linearly independent components on a neighborhood of 0 such that $f(0)=0$ and $h(0)=0$. Let us assume the regular observability rank condition ${ }^{1}$. More precisely, if $h=$ $\left(h_{i}\right)_{1 \leq i \leq m}$ then, as for the linear case, there exist integers $r_{1} \geq r_{2} \ldots r_{m}$ (for more details see for example [7]) such that: $\quad \operatorname{rank}\left\{\mathrm{dh}_{1}, d L_{f} h_{1}, . ., d L_{f}^{r_{1}-1} h_{1}, . ., d h_{m}, . ., d L_{f}^{r_{m}-1} h_{1}\right\}=$ $m$. In [11], we can fund corresponding vector fields $\left\{\tau_{i, 1}\right\}_{1 \leq i \leq m}$ by the same equations as in (19-20). Thus: equations :

$$
\begin{array}{r}
d L_{f}^{k-1} h_{j}\left(\tau_{i, 1}\right)=0 \text { for } j<i \text { and } 1 \leq k \leq r_{i} \\
d L_{f}^{k-1} h_{i}\left(\tau_{i, 1}\right)=0 \text { for } 1 \leq k \leq r_{i} \\
d L_{f}^{r_{i}-1} h_{i}\left(\tau_{i, 1}\right)=1 \\
d L_{f}^{k-1}{ }_{j} \tau_{i, 1}=0 \text { for } j>i \text { and } 1 \leq k \leq r_{j} .
\end{array}
$$

\footnotetext{
${ }^{1}$ locally weakly observable without singularity, i.e. fixe observability index
}

And then, we define:

$$
\tau_{i, j}=\left[\tau_{i, j+1}, f\right] \quad \text { for } 1 \leq j \leq r_{i}-1 .
$$

where the [,] means the Lie bracket. Now, the main difference between the nonlinear and the linear case is that for the nonlinear case, we assume in lemma 1 and corollary 2 that vector fields $\tau_{i, j}$ commute:

$$
\left[\tau_{i, j}, \tau_{k, s}\right]=0
$$

This last condition is always fulfilled in the linear case because the vector fields $\tau_{i, j}$ are constant. Now, for condition (23) is the same for both case linear one and nonlinear one. To summer, the important thing to keep in mind is:

- Condition (26), which is always fulfilled in the linear case, ensures the existence of the coordinate change in which the dynamic is in form (5),

- Condition (23), ensures that the outputs in the new coordinates are only functions of the outputs in the former coordinates.

To end this discussion, we can say that the corollary (1) provide a necessary condition for the multi-output nonlinear dynamical systems. Indeed, if we set:

$$
\begin{aligned}
A & =\frac{\partial f}{\partial x}(0) \text { itslinearpartat } 0 \\
C & =\frac{\partial h}{\partial x}(0) \text { itslinearpartat } 0
\end{aligned}
$$

then, the existence of change of coordinates which transforms the nonlinear dynamical system (24-25) into the canonical form (5-6) implies the existence of a change of coordinates which transforms the linear part $(A, C)$ into the canonical form (5-6). Obviously, it is only a necessary condition, because this does not guarantee that the linear part is only function of $y$ and $u$.

\section{CONCLUSION}

This paper dealt with the Brunovsky observability normal form for a multi-output linear dynamical system. Then, it gave a comparison between with the stated result and the well-known results in the nonlinear case.

\section{REFERENCES}

[1] D. Boutat, G. Zheng, J.P. Barbot, and H. Hammouri. Observer error linearization multi-output depending. IEEE CDC, 3:199216, 2006.

[2] D.Boutat, A. Benali, and H. Hammouri. Geometrical conditions for observer error linearization linearization with a diffeomorphism on the outputs. Nolcos 2007, 3: 199216, 2007.

[3] P. Brunovsky. A classification of linear controllable sys- temd. Keybernetika, 3(6):173188, 1970.

[4] M. Hou and A.C. Pugh. Observer with linear error dynamics for nonlinear multi-output systems. Systems and Control Letters, 37:19, 1999.

[5] R.E. Kalman and R. Bucy. A new results in linear Fltering and prediction theory. Transcation of ASME, Journal of Basic Engineering,, $82: 3540,1960$.

[6] A.J. Krener and A. Isidori. Linearization by output injection and nonlinear observer. System and Control Letters, 11:4752, 1983.

[7] A.J. Krener and W. Respondek. Nonlinear observer with linearizable error dynamics. SIAM J. Control and Optimization, 30:197216, 1985.

[8] D.G. Luenberger. An introduction to observers. IEEE Transactions on Automatic Control, 16, 6:596602, Dec, 1971. 
[9] R. Marino and P. Tomei. Nonlinear Control Design : Geometric, Adaptive abd Robust. Prentice Hall, 1995. D. Noh, N.H. Jo, and H. Seo. Nonlinear observer design by dynamic observer error linearization. IEEE Trans- actions on Automatica Control, 49, 10, 2004.

[10] A.R. Phelps. On constructing nonlinear observers. SIAM J. Control and Optimization, 29, 1991. F. Plestan and A. Glumineau. Linearization by generalized input output injection. Syst. Contr. Letters, 31:115128, 1997.

[11] X.H. Xia and W.B. Gao. Nonlinear observer with lineariz- able error dynamics. SI 